\title{
Actores sociales y políticas públicas en el desarrollo rural
}

Eduardo Moyano y María del Mar Giménez Guerrero*

En el marco de la UE no puede hablarse todavía de la existencia de una política europea de desarrollo rural formulada desde las instituciones comunitarias y que haya generado un conjunto normativo similar al de la PAC (Política Agraria Común). Lo que hay son acciones de tipo experimental, limitadas a ámbitos territoriales específicos y adoptadas o bien como iniciativas de la propia Comisión Europea, que las financia con cargo a su propio presupuesto -como ocurre con el programa LEADER-, o bien dentro de planes de desarrollo regional -como ocurre con el reciente programa PRODER.

La adopción de estas iniciativas a principios de los años noventa respondía al cambio de filosofía introducida en el discurso de la Comisión a raíz de la publicación del "Informe sobre el Futuro del Mundo Rural" (1988), cambio que venía a situar un contrapunto en los intensos debates desarrollados por aquellos años sobre la necesidad de recortar el gasto agrícola, modificar los mecanismos de protección basados en la política de precios y abordar una reforma en profundidad de la PAC. Lejos de las preocupaciones de tipo más coyuntural que rodeaban a la reforma de la PAC, como eran las de un presupuesto descontrolado y unos excedentes agrícolas desorbitantes, el citado Informe hacía un análisis reposado sobre los problemas del mundo rural en el marco de las sociedades industriales avanzadas, procurando recoger los cambios que se estaban ya dando en la opinión pública europea y que demandaban un nuevo modelo de desarrollo.

Además de las iniciativas LEADER, el debate abierto en la sociedad europea sobre la necesidad de abordar los problemas del mundo rural desde políticas diferentes de las estrictamente relacionadas con la produción agrícola, aunque no en contraposición con ellas, también ha significado desde entonces la adopción, por parte de los gobiernos nacionales y regionales, de políticas propias de desarrollo rural en las que el poder público asume un pa-

\footnotetext{
* IESA-CSIC de Andalucía. Córdoba.
} 
pel dinamizador, pero no directamente intervencionista, participando en la financiación y regulación de las iniciativas procedentes de la propia sociedad rural. Por lo general, estas políticas regionales y/o nacionales de desarrollo rural han sido formuladas en países en los que se dan importantes hándicaps estructurales en sus zonas rurales, viviendo la población de estas áreas en condiciones de peor calidad que las poblaciones de las áreas urbanas.

La gradual reforma que se ha ido introduciendo en la política agraria de la UE desde mediados de los años 80 -y culminada con el paquete de medidas aprobado en 1992- ha supuesto una paulatina reducción de los niveles de protección vía precios de garantía y una creciente liberalización de los mercados agrícolas. Todo ello ha hecho más vulnerables las explotaciones agrarias a la competencia y ha abierto un horizonte de mayor incertidumbre entre los agricultores, si bien mitigada en estos útimos cuatro años por el colchón -coyuntural y en absoluto asegurado para el futuro- que han significado las ayudas directas de superficie en algunos importantes sectores como los cereales. Las reformas, pendientes todavía de aprobar o desarrollar, de algunas OCMs -como las del aceite de oliva, el vino o las del sector hortofrutícola- de gran importancia para muchas regiones rurales europeas, introducen elementos adicionales de incertidumbre en el desarrollo económico de estas zonas, lo que explica que cada vez se vaya abriendo paso la idea de que su futuro debiera plantearse desde perspectivas más amplias que las meramente relacionadas con la actividad agraria.

En este contexto emergen las nuevas políticas públicas en materia de desarrollo rural, políticas que se plantean de acuerdo con lógicas diferentes a las que han inspirado hasta ahora las políticas agrarias. Algunas de estas nuevas políticas, formuladas por iniciativa de los gobiernos nacionales o regionales en el marco de sus correspondientes planes de desarrollo -como es el caso del Plan de Desarrollo Rural de Andalucía- procuran ajustarse a los criterios establecidos por la Comisión Europea, de modo que puedan ser homologadas y recibir así algún tipo de financiación adicional. Todo este paquete de iniciativas, surgido tanto desde la Comisión Europea como de los propios gobiernos nacionales o regionales y avalado institucionalmente por la conferencia celebrada el pasado mes de noviembre en la ciudad irlandesa de Cork -conferencia presidida por el propio comisario de Agricultura Fischler-, permite que se pueda ya hablar del comienzo de una política específica de desarrollo rufal, diferenciada de la política agraria. Aunque todavía en fase embrionaria, creemos que las distintas iniciativas en materia de desarrollo rural guardan entre sí una serie de rasgos comunes cuyo análisis nos puede orientar sabre la naturaleza, objetivos e instrumentación de la futura política. 


\section{Algunos elementos comunes a las políticas de desarrollo rural}

Aunque sólo los actuaciones que se desarrollan dentro de la iniciativa LEADER o del programa operativo PRODER tienen que cumplir una serie de requisitos de carácter general, la realidad es que la mayor parte de las políticas de desarrollo rural coinciden en algunos elementos comunes que responden al hecho de inspirarse en una misma filosofía y tener que afrontar problemas muy similares. En este apartado mencionaremos algunos de esos elementos ${ }^{1}$.

\section{Principios y filosofía}

Las políticas de desarrollo rural se caracterizan por compartir una misma filosofía, que refleja los cambios producidos en el marco de referencia en el que se habían situado hasta sólo unos años los problemas de la agricultura y el mundo rural. Estos cambios responden no sólo a factores económicos, sino también de índole política y cultural. El logro de la autosuficiencia alimentaria por parte de los países industrializados, el avance de la conciencia ecológica entre la opinión pública o la necesidad de liberalizar los mercados internacionales para facilitar los intercambios comerciales, son algunos de los factores que explican que el marco de referencia de la agricultura y el mundo rural haya cambiado de forma sustancial en los últimos años.

Las políticas de desarrollo rural parten del reconocimiento de que hoy día no es posible acercarse a los problemas del mundo rural con el mismo bagaje intelectual que se utilizaba al comienzo de los años ochenta, admitiendo que el objetivo prioritario de intensificar la producción agrícola ha sido sustituido por la conveniencia de introducir prácticas extensivas y de forestación capaces de devolver a muchos espacios naturales su vocación forestal y evitar los riesgos de desertificación que asolan a algunas regiones europeas. Asimismo, la visión agrarista del desarrollo, que identificaba desarrollo rural con desarrollo agrícola, ha sido sustituida en los nuevos planes por una concepción más integral en la que se plantean estrategias capaces de dinamizar todos los recursos endógenos -agrícolas y no agrícolas- existentes en las comunidades rurales.

Es en este nuevo marco de referencia en el que las nuevas políticas de desarrollo rural plantean los problemas del mundo rural, aceptando como un hecho indudable que la resolución de los mismos no puede descansar sólo y exclusivamente sobre las espaldas de un sector agrario cada vez más redimensionado con criterios de competitividad, sino que se

${ }^{1}$ Este apartado toma como referencia el Plan de Desarrollo Rural de Andalucía, si bien el análisis se extiende a otros planes consultados por los autores. 
hace necesario diversificar las actividades productivas impulsando cualquier iniciativa viable de desarrollo. El escenario de partida de estas nuevas políticas es el de un mundo rural en el que la agricultura, aunque continúe siendo una actividad central en términos económicos, crea cada vez menos empleo, planteando por ello la necesidad de que se generen nuevas actividades productivas para absorber la mano de obra existente, dado que la tradicional vía de escape a los sectores industrializados urbanos ya no es viable como consecuencia de la crisis del modelo de desarrollo fordista y la finalización de las políticas de pleno empleo.

\section{2.- Objetivos}

Los objetivos que suelen establecerse en las políticas de desarrollo rural son los siguientes: a) la generación de empleo en el medio rural, b) el aumento del nivel de renta de su población, y c) la mejora de sus condiciones de vida y de trabajo. Todo ello con la finalidad última de mantener un nivel equilibrado de población en el marco de la sociedad rural y propiciar una integración más armónica entre los mundos rural y urbano.

Un elemento fundamental de la filosofía que subyace a las políticas de desarrollo rural es el firme convencimiento de que los objetivos anteriores sólo podrán lograrse con la participación activa de los diferentes agentes económicos y sociales que existen en el mundo rural, y que deberán alcanzarse teniendo en cuenta las exigencias planteadas por el desarrollo sostenible, dada la especial importancia que el medio rural posee en relación con la proticción y conservación del medio ambiente.

En suma, las políticas de desarrollo rural, materializadas en los correspondientes planes y programas, se configuran como un impulso de dinamización del mundo rural que haga más eficientes sus estructuras productivas y mejore la calidad de vida de su población y de la sociedad en su conjunto.

\section{3.- Líneas estratégicas y políticas de actuación}

Las líneas estratégicas muestran la forma de alcanzar los objetivos de la política de desarrollo rural a través de las correspondientes políticas sectoriales. Tales líneas suelen girar en torno a tres grandes ejes: I) movilización social y cultural de la sociedad rural; II) diversificación y modernización de las actividades económicas, y III) equidad territorial. No en todos los casos, estos ejes están presentes en las políticas de desarrollo rural, dependiendo de la naturaleza de los planes y programas que se ponen en marcha. 
Normalmente son los dos primeros ejes los que suelen estar presentes en todos los programas de desarrollo rural -del tipo de los programas LEADER o PRODER mencionados-, mientras que el tercer eje -el de la equidad territorial-, acompaña a los otros dos cuando se formulan planes articulados en los que se implican directamente los poderes públicos -como es el caso del Plan de Desarrollo Rural de Andalucía. A continuación se analizarán cada uno de estos ejes.

\section{Eje I) MOVILIZACIÓN SOCIAL Y CULTURAL DE LA SOCIEDAD RURAL}

Como es conocido, el objetivo básico de generar empleo y elevar el nivel de renta de la población no sólo guarda relación con el ámbito productivo, sino que afecta también a los ámbitos social y cultural. Por ello, las políticas de desarrollo rural suelen plantear la necesidad de una estrategia de movilización que ayude a impulsar las transformaciones que conlleva unas sociedades en mutación como son las sociedades rurales de hoy. Este empeño por crear un clima favorable que posibilite la movilización de la sociedad rural y la emergencia dè nuevos proyectos económicos es lo que mueve a las políticas de desarrollo rural a proponer en sus planes y programas tres tipos de políticas específicas de actuación. A saber: una política de dinamización social, una política de formación y cualificación profesional, y una política de conservación del patrimonio cultural.

\section{a) Política de dinamización social}

El eje básico de esta política suele ser el fomento de "grupos de desarrollo rural", constituidos por la propia población y reconocidos como ejes fundamentales en la presentación y posterior desarrollo de las iniciativas. De acuerdo con la filosofía que subyace a los políticas de desarrollo rural, estos grupos -que reciben distintas denominaciones según los casos y que en los programas LEADER se denominan, por ejemplo, "grupos de acción local"- están destinados a convertirse en el marco de colaboración adecuado para que puedan actuar de forma coordinada los diferentes entes administrativos, las empresas privadas, las organizaciones profesionales y cooperativas, los sindicatos, los grupos de interés económico y, en general, todos los agentes socioeconómicos que se preocupen por el aumento del nivel de renta, la generación de empleo en el medio rural y la mejora del bienestar de su población. Los Grupos dan expresión organizativa a todos esos agentes socioeconómicos procurando no excluir ningún tipo de iniciativa y dotarse de la estructura más idónea para el ejercicio de su actividad -en unos casos, el grupo adquiere la configuración de una sociedad cooperativa, mientras que, en otros, optan por constituir una fundación, no faltando los que se inclinan por la fórmula de sociedad anónima.

Experiencias ya existentes en el conjunto de la UE han puesto de manifiesto que las iniciativas de desarrollo rural más prósperas han sido aquéllas en las que han actuado de 
forma conjunta este tipo de grupos intermedios. En dichas experiencias, los "grupos de desarrollo rural" han demostrado, actuando conjuntamente, tener gran capacidad para conseguir fuentes de financiación a proyectos viables de desarrollo y para influir ante los poderes públicos en la dotación a las comunidades rurales de los equipamientos y servicios necesarios para consolidar su tejido económico, aspectos éstos que difícilmente pueden lograrse a través de demandas dispersas e inconexas procedentes del ámbito local.

\section{b) La política de formación y cualificación profesional}

Paralelamente a las tareas de dinamización social, las políticas de desarrollo rural dedican una atención especial a la formación y cualificación profesional, ya que entienden que poseer una adecuada formación profesional y disponer de mano de obra con actitud positiva hacia el trabajo, constituyen dos factores de primera magnitud en la creación de nuevas oportunidades de empleo y desarrollo económico. Por ello, suelen instar a las entidades públicas con responsabilidad en el terreno educativo a la adaptación de la oferta educativa a los profundos y veloces cambios que se vienen produciendo en nuestros días y, en concreto, a los importantes desafíos que ha de afrontar el mundo rural europeo.

Igualmente, consideran necesario ampliar dicha oferta formativa, a fin de que alcance al máximo de la población residente en el medio rural, toda vez que, como se ha señalado anteriormente, la mayor cualificación del capital humano constituye el mejor instrumento de futuro para el desarrollo rural. En este sentido, las políticas de desarrollo rural apuestan claramente por una acción decidida en las líneas de formación en materia de gestión e iniciativa empresariales, proponiendo actuaciones específicas en la formación profesional reglada y ocupacional, así como en la formación continua.

\section{c) La política de conservación del patrimonio cultural}

Entendiendo el patrimonio cultural en un sentido amplio, es decir, incluyendo elementos de naturaleza intangible como son los diferentes estilos de vida y costumbres, el folklore o la gastronomía, las políticas de desarrollo rural suelen proponer acciones específicas para concienciar a la población sobre la necesidad de mantener y mejorar la calidad de los asentamientos rurales -al ser una de las principales señas de identidad regional- y sobre el valor que tiene el patrimonio rural como recurso propio de cada región. De este modo, se pretende poner énfasis en la importancia de la autoestima para potenciar los valores propios de una región y consolidar sus señas de identidad. En esta líneá, las planes y programas suelen valorar muy positivamente las experiencias de las Escuelas-Taller en la recuperación del patrimonio arquitectónico de las comunidades rurales, estableciendo módulos especializados en los distintos oficios relacionados con la construcción rural. 


\section{Eje II) DIVERSIFICACIÓN Y MODERNIZACIÓN DE LAS ACTIVIDADES ECONÓMICAS}

Dada la insuficiente capacidad del sector agrario para generar el nivel de empleo que sería necesario para resolver el problema del desempleo en muchas zonas rurales, las políticas de desarrollo rural consideran como necesaria una estrategia de diversificación productiva que aproveche al máximo los recursos infrautilizados u ociosos de cada región. Para ello, prevén una serie de actuaciones articuladas en torno a las correspondientes políticas sectoriales, cuyos rasgos fundamentales expondré a continuación.

\section{a) Política agraria}

Aunque parten del reconocimiento de que la agricultura tiene que complementarse con otras actividades productivas, las políticas de desarrollo rural no olvidan la importancia que para el desarrollo de las comunidades rurales tiene la existencia de una agricultura moderna. Por ello, suelen incluirse actuaciones específicas en el terreno de la política agraria. Así, es frecuente que los planes de desarrollo rural incluyan una política sectorial destinada a impulsar la modernización y mejora de las explotaciones agrícolas y ganaderas, potenciando las actuaciones que favorezcan la diversificación de los cultivos y la mejor comercialización de los productos agrarios. En este sentido algunos planes proponen incluso la elaboración de un programa de modernización agraria que contemple, entre otras cosas, la mejora de las infraestructuras de apoyo a las explotaciones, la incorporación de los jóvenes a la actividad agraria o el fomento de la agricultura de grupo. En esa misma línea de impulso a la modernización agraria, se propone la mejora de las superficies equipadas para riego, la mejora de la sanidad animal y vegetal y la potenciación del sistema de seguros agrarios.

El fomento y mejora de las organizaciones de productores, así como la promoción de la agricultura contractual y de las organizaciones interprofesionales, son considerados por los planes de desarrollo rural como elementos fundamentales para que la modernización afecte también al ámbito de la comercialización y transformación facilitando una mejor integración entre los sectores de la producción y de la industria agroalimentaria.

\section{b) Política industrial}

Atendiendo a la propia contribución de la cultura industrial en el proceso de modernización de la sociedad, las políticas de desarrollo rural consideran que el futuro desarrollo del mundo rural no puede plantearse al margen de una base industrial amplia y diversificada, sino todo lo contrario, construyéndola a partir de la incorporación de nuevas activida- 
des y de la modernización competitiva de las ya establecidas. En función de estos objetivos, en el marco de dichas políticas de desarrollo rural se suele fomentar la instalación de industrias en el medio rural y la modernización empresarial y tecnológica promoviendo la formación de un entorno tecnológico y de prestación de servicios avanzados a la producción en el medio rural, así como se suele fomentar también una mejor adecuación de la investigación y desarrollo $(\mathrm{I}+\mathrm{D})$ a los requerimientos industriales en el medio rural.

También las políticas de desarrollo rural suelen apoyar proyectos destinados a la potenciación de la transferencia de tecnologías, mediante instrumentos que canalicen la información de los resultados de los investigadores y las necesidades de innovación de las empresas, con el fin de incorporar al tejido productivo los descubrimientos científicos e innovaciones básicas necesarias para que las empresas instaladas en el medio rural puedan incorporar dichos conocimientos en sus actividades productivas.

El fomento de las energías renovables, apoyando la generación de energía (eléctrica o térmica) a partir de las mismas (biomasa, energía hidraúlica o térmica), es otra de las prioridades de las políticas de desarrollo rural en materia industrial. En ese sentido, promueven la utilización de recursos renovables en la producción de energía a través de la instalación y rehabilitación de centrales minihidráulicas, la promoción de sistemas solares térmicos o de instalaciones de gas natural y combustibles biomásicos.

\section{c) Política forestal}

Las políticas de desarrollo rural suelen fijarse como fin primordial hacer compatible la explotación productiva de los bosques con su regeneración natural, así como con el disfrute de los mismos como zonas de esparcimiento, ocio y actividades deportivas. En este sentido se considera que los espacios forestales merecen una atención especial tanto como generadores de rentas procedentes de diversas actividades (aprovechamiento de la madera, pastos, caza, pesca, corcho, esencias, frutos), como por la importancia que están adquiriendo en relación con las actividades de ocio y turismo, sin olvidar la función ambiental global que desempeñan.

En coherencia con estos objetivos, las políticas de desarrollo rural se plantean líneas de actuación como las siguientes: a) promover la implicación de la sociedad rural en el aprovechamiento y gestión de los espacios forestales, mediante la incorporación concertada de la iniciativa privada en el uso de montes y espacios públicos forestales; b) impulsar la conservación y restauración del patrimonio forestal como estrategia de fomento del turismo rural en sus diversas modalidades; c) desarrollar la planificación de las zonas forestales, haciendo una gestión integrada de los ecosistemas que las constituyen, mediante ac- 
tuaciones sobre la vegetación -como la repoblación forestal, incorporando criterios de calidad visual del paisaje- $y$ actuaciones en materia de incendios, tanto las referentes a su prevención, como a su extinción y posterior repoblación de las tierras afectadas por los mismos; d) acometer la ordenación y fomento de las actividades cinegéticas y piscícolas, dando prioridad a las actuaciones tendentes a la recuperación y repoblación de las diferentes especies de caza, mejorando el estado sanitario de las mismas.

\section{d) Política de turismo rural}

La promoción del turismo en el medio rural es considerada por las políticas de desarrollo rural una de las oportunidades más destacadas entre las diversas alternativas de diversificación productiva. Ahora bien, también suelen señalar que el turismo, a pesar del interés indudable que tiene, no puede ser la solución general para todos los espacios rurales, ni debe ser tratado de la misma manera que en las zonas de litoral, en donde la masificación fue la clave del modelo de explotación.

Las políticas de desarrollo rural suelen considerar que la oferta de un producto específico de calidad, que no se limite a la mera restauración y alojamiento, sino que aproveche los recursos naturales y paisajísticos existentes y la riqueza del patrimonio rural y cultural, constituye una vía de diferenciación para el turismo rural. Asimismo, consideran que el turismo rural debe ser contemplado como una oportunidad de generar nuevas rentas para los agricultores en un momento como el actual en que deben afrontar un proceso de cambio acelerado. Pero, como se señala, la oferta turística debe contemplar un producto singular y completo, integrando en el mismo un conjunto consistente y cualificado de actividades de ocio y turismo. De este modo, muchos planes y programas de desarrollo rural proponen que se considere el producto turístico de una forma integral, esto es, como la suma de infraestructuras, servicios a empresas, tecnologías y recursos humanos, que producen bienes susceptibles de ser consumidos por el turista.

\section{e) Política de medio ambiente}

Las políticas de desarrollo rural ponen un énfasis especial en la valorización de los recursos medioambientales como un componente fundamental de toda estrategia de desarrollo. Por ello, suelen considerar que alcanzar el equilibrio entre desarrollo económico y protección del medio ambiente supone, pues, tener en cuenta las consideraciones ecológicas a la hora de formular y aplicar las distintas políticas económicas y sectoriales. En este sentido, consideran que debe incrementarse en la sociedad la sensibilidad acerca de la importancia de los recursos medioambientales y de la potencialidad que los mismos poseen para crear nuevas actividades generadoras de renta y empleo. 
Las líneas de actuación que se suelen proponer en materia medioambiental van dirigidas a aspectos como los siguientes: a) mejora de la calidad de las aguas continentales, regenerando los impactos contaminantes de las aguas residuales urbanas y mejorando la salubridad y la calidad ambiental de las distintas redes de abastecimiento en el medio rural; b) mejora de la gestión y control de residuos, realizando campañas de divulgación e información entre la población rural sobre los efectos de la generación excesiva de residuos sobre el medio ambiente, sobre la conveniencia de un menor consumo de materiales contaminantes y sobre la necesidad de tener un comportamiento responsable con los residuos generados; c) conservación de suelos, priorizando la lucha contra la erosión y desestabilización de suelos dentro de las acciones regenerativas del medio ambiente; d) protección de la flora y la fauna, mediante la realización de inventarios, censos, proyectos de recuperación de especies endémicas y amenazadas, o actuaciones destinadas a la sensibilización ciudadana sobre el valor del patrimonio en este campo; e) mejora de la gestión de los espacios naturales protegidos, favoreciendo su conocimiento y disfrute, y potenciando todas las actividades económicas relacionadas con sus recursos endógenos y que sean compatibles con las exigencias medioambientales de estos espacios; f) desarrollo sostenible en los parques naturales y sus áreas de influencia socioeconómica, fundamentalmente mediante la puesta en marcha de planes de desarrollo integral, incentivando y apoyando las actividades que sean compatibles con la conservación (prácticas agrarias ecológicas; obtención de esencias, leña y carbón vegetal; actividades cinegéticas y piscícolas, turismo verde, etc); g) conservación, regeneración y mejora del paisaje, protegiendo aquellos elementos que presentan valores estéticos, ecológicos y culturales, restaurando las zonas que hayan sufrido degradación, introduciendo elementos innovadores que contribuyan a la creación de un paisaje de mayor valor ecológico o visual, y adoptando criterios encaminados a lograr la integración armónica en el paisaje de las actuaciones públicas o privadas que hayan de llevarse a cabo.

\section{Eje III) EQUIDAD TERRITORIAL}

Cuando las políticas de desarrollo rural se plantean de modo articulado y con un objetivo de actuación integral en el medio rural y no sólo como simples programas sectoriales de actuación, suelen incluir un tercer eje -equidad territorial-, en el que el papel del Estado adquiere un protagonismo que no existe en los dos ejes anteriores. Por lo general, las politicas de desarrollo rural parten del reconocimiento de que la lógica del mercado genera desigualdades que deben ser identificadas y corregidas por los poderes públicos. Consideran, por tanto, necesario que los poderes públicos actúen de un modo específico para corregir dicha tendencia, favoreciendo un mayor potencial de crecimiento económico (dotación de infraestructuras básicas o incentivos para la promoción económica), así como mejorando el nivel de los equipamientos sociales en las zonas más atrasadas. 
En el caso de regiones con una mayor heterogeneidad estructural con respecto a otras regiones europeas, se hace aún más justificada la necesidad de la intervención pública para compensar los desequilibrios generados por la propia lógica del mercado. Por ello, la mayor parte de las políticas de desarrollo rural suelen contener una estrategia explícita de equidad territorial, cuya finalidad es atenuar los desequilibrios ocasionados por lá dinámica del mercado y mejorar la calidad de vida de la población que habita en las comunidades rurales. Las actuaciones públicas dirigidas a incrementar las dotaciones de infraestructuras básicas y equipamientos sociales en las comunidades rurales más desfavorecidas constituyen aspectos decisivos de esta estrategia de equidad territorial.

Igualmente, dicha estrategia de equidad planteada en las políticas de desarrollo rural trata de establecer la correspondiente discriminación positiva en favor de las áreas rurales más desfavorecidas en lo relativo a los servicios de educación, formación profesional y capacitación empresarial, con objeto de nivelar las diferencias respecto de otras áreas y aumentar sus posibilidades para descubrir y promover iniciativas de carácter productivo a partir de sus propios recursos. Este tipo de estrategia no está guiada exclusivamente por motivaciones asistencialistas, ya que la política de equidad territorial es, al mismo tiempo, una política orientada a mantener en las comunidades rurales un nivel de población suficiente para hacer viables las iniciativas productivas que se promuevan.

Las acciones en materia de mejora de las infraestructuras rurales, de equipamientos y servicios colectivos y de servicios sociales, constituyen los ejes básicos de la política de equidad territorial que suelen establecer las políticas públicas de desarrollo rural para compensar los desequilibrios existentes en el seno de la sociedad rural.

\section{4.- Coordinación administrativa de las política de desarrollo rural}

Cuando se elaboran las políticas de desarrollo rural suele tenerse en cuenta que las distintas líneas estratégicas y actuaciones sectoriales no serían posibles sin la necesaria coordinación de las administraciones que inciden en el medio rural, tomándose conciencia de los problemas suscitados por la multiplicidad de agentes que intervienen en los temas de desarrollo y por la frecuente superposición de sus respectivas competencias. En este sentido, la vocación de las políticas de desarrollo rural, plasmadas en los correspondientes planes y programas, es la de evitar que se conviertan en una simple superposición de políticas horizontales y de actuaciones sectoriales; con este propósito introducen una concepción nueva del desarrollo, una concepción de carácter integral y participariva.

No obstante, estas políticas tienen en cuenta que, en el medio rural, distintas administraciones públicas (central, regional, local y de la UE) tienen responsabilidades y compe- 
tencias compartidas en el desarrollo rural, al poseer instrumentos de política social y económica con incidencia sobre la realidad rural. Por ello, para que las actuaciones de las distintas administraciones públicas que operan en las zonas rurales resulten eficaces, las políticas de desarrollo rural consideran necesario poner en marcha mecanismos de coordinación que garanticen la coherencia de las respectivas políticas.

De ahí que las políticas públicas de desarrollo rural se conciban como planes coordinados en la medida en que participan en su aplicación diversos poderes públicos, mejorando el diseño y variedad de los programas a ejecutar: unos, recogidos en el correspondiente plan de desarrollo rural, y otros, recogidos en los diversos documentos de programación socioeconómica que las administraciones públicas interesadas habrán de elaborar. De ahí que muchos planes hayan posibilitado la creación de agencias interdepartamentales para garantizar la necesaria coordinación entre las distintas administraciones, venciendo la tentación agrarista de asignar la canalización de su aplicación al ministerio de Agricultura.

\section{Desarrollo rural y actores sociales}

La elaboración de planes y programas de desarrollo rural, bien con carácter experimental y restringidos a zonas muy concretas -como es el caso de los programas LEADER-, o bien con carácter de más largo plazo y cubriendo el territorio de una región -como son los aprobados por gobiernos nacionales o regionales en los correspondientes planes de desarrollo-, representan la emergencia de nuevas políticas públicas para responder a los efectos producidos por las profundas transformaciones experimentadas en los modelos de desarrollo económico. Lejos de las previsiones de que los procesos de liberalización de los mercados y de globalización conducen a una retirada generalizada del Estado de la economía y al retorno de la sociedad civil, parece más sensato pensar que tales procesos provocan efectivamente la retirada del Estado de algunas áreas, pero también su presencia en otras nuevas necesitadas de la función estatal reguladora y de la aplicación de principios de interés general.

En estas nuevas áreas, entre las que podemos situar las del desarrollo rural, la presencia del Estado se acompaña de la emergencia de nuevos actores sociales -como son los grupos de desarrollo rural-, que representan a una sociedad plural y diversificada en la que los agricultores ya no son el grupo social dominante, sino una minoría entre otras, un grupo social como otros que debe compartir en posición de igualdad los problemas del mundo rural y la búsqueda de soluciones. La integración de los agricultores en las iniciativas de desarrollo rural tal vez sea uno de los retos que tienen planteadas muchas zonas rurales europeas en las que la población agrícola todavía es importante; de su integración en esas iniciativas depende que los agricultores salgan del gueto en que los discursos agraristas le habían metido bajo la seducción de que formaban parte de un mundo singular y, por ello, me- 
recedor de un tratamiento privilegiado. La apertura de los mercados agrícolas y la reforma de los mecanismos de protección colocan a los agricultores en una posición real y no ficticia, una posición como la de cualquier otro grupo socioprofesional que ha de buscar fuentes complementarias de rentas y apoyar la creación de nuevas riquezas en el mundo rural a través de la diversificación de las actividades productivas.

Las políticas de desarrollo rural plantean también el reto de la emergencia de nuevas agencias estatales capaces de coordinar las distintas actuaciones administrativas y de crear marcos adecuados de concertación con los actores sociales y económicos implicados. La tentación de canalizar las políticas de desarrollo rural a través de los ministerios de Agricultura ha generado problemas de coordinación y legitimidad, dado que son políticas cuya filosofía subyacente es la de que una agricultura modernizada no puede por sí sola generar el empleo ni la riqueza necesaria para el desarrollo de las zonas rurales, sino que se hace necesario movilizar todos los recursos disponibles para afrontar los problemas de estas zonas desde una perspectiva integral. La creación de agencias interministeriales parecen soluciones más adecuadas para esa necesaria coordinación entre las diversas entidades públicas.

En definitiva, la política de desarrollo rural comienza a desarrollarse como una política diferenciada de la PAC, integrando de manera global los problemas del mundo rural y respondiendo a las nuevas demandas de una sociedad como la europea que, al haber alcanzado la autosuficiencia alimentaria y unos niveles elevados de bienestar, se plantea ahora cómo hacer viable la continuidad del modelo equilibrado entre campo y ciudad que ha sido uno de los grandes patrimonios de la cultura europea y uno de los elementos básicos de su cohesión social. La política de desarrollo rural tiende precisamente a evitar que los efectos no queridos del mercado acaben por romper ese equilibrio territorial, y para ello pretende ser un instrumento para dinamizar las iniciativas económicas y garantizar la equidad en las zonas menos favorecidas. 